Draft VERsion November 9, 2018

Preprint typeset using $\mathrm{LAT}_{\mathrm{E}} \mathrm{X}$ style emulateapj v. 5/2/11

\title{
BLACK HOLE MAGNETOSPHERES
}

\author{
Antonios Nathanail ${ }^{1,2, *}$ and IoAnNis Contopoulos ${ }^{1}$ \\ 1 Research Center for Astronomy and Applied Mathematics, Academy of Athens, Athens 11527, Greece \\ and \\ ${ }^{2}$ Section of Astrophysics, Astronomy and Mechanics, Department of Physics, University of Athens, \\ Panepistimiopolis Zografos, Athens 15783, Greece \\ Draft version November 9, 2018
}

\begin{abstract}
We investigate the structure of the steady-state force-free magnetosphere around a Kerr black hole in various astrophysical settings. The solution $\Psi(r, \theta)$ depends on the distributions of the magnetic field line angular velocity $\omega(\Psi)$ and the poloidal electric current $I(\Psi)$. These are obtained self-consistently as eigenfunctions that allow the solution to smoothly cross the two singular surfaces of the problem, the Inner Light Surface (ILS) inside the ergosphere, and the Outer Light Surface (OLS), which is the generalization of the pulsar light cylinder. Magnetic field configurations that cross both singular surfaces (e.g. monopole, paraboloidal) are uniquely determined. Configurations that cross only one light surface (e.g. the artificial case of a rotating black hole embedded in a vertical magnetic field) are degenerate. We show that, similarly to pulsars, black hole magnetospheres naturally develop an electric current sheet that potentially plays a very important role in the dissipation of black hole rotational energy and in the emission of high-energy radiation.
\end{abstract}

Subject headings: Accretion; Black hole physics; Magnetic fields

\section{INTRODUCTION}

Several types of powerful high-energy sources exist in the universe (X-ray binaries, gamma-ray bursts, active galactic nuclei, etc.). In many of these systems the central engine is believed to involve a rotating black hole threaded by large scale astrophysical magnetic fields. It has been shown theoretically that a rotating black hole can radiate away its available reducible energy through some kind of generalized Penrose process (e.g. Lasota et al. 2014). Blandford \& Znajek (1977) (hereafter BZ) discussed one particular such process where the magnetic field taps the rotational energy of the black hole and generates powerful outflows of electromagnetic (Poynting) energy. They argued that space-time frame-dragging induces an electric field that is strong enough to 'break' the vacuum and establish an electron-positron force-free magnetosphere. They also obtained the structure of this magnetosphere for simple monopole and paraboloidal boundary conditions, and estimated the flux of electromagnetic energy for low black hole spin parameters.

A few years later, MacDonald \& Thorne (1982) investigated the same problem in the ' $3+1$ ' formulation. Their approach gave the opportunity for astrophysicists not familiar with the geometrical language of general relativity to enter the field of black hole magnetospheres and bring with them their expertise from other areas of astrophysics (e.g. pulsar research). In their formulation all physical quantities are measured by Zero Angular Momentum Observers (ZAMOs; also known as local Fiducial Observers or Fidos). In their Locally Non-Rotating Frame (LNRF) the equations for the electromagnetic field are very-similar in form to their respective equations in flat spacetime. Furthermore, Thorne, Price \& MacDonald (1986) introduced the so called 'membrane paradigm' where they argued that the key element of the

*antonionitoni@hotmail.com
BZ mechanism is the (stretched) black hole horizon.

Punsly \& Coroniti (1990) proposed a different perspective based on a magnetohydrodynamic (MHD) model. A few years later Komissarov (2001, 2004a,b) performed general relativistic MHD numerical simulations and found that the BZ monopole solution is aymptotically stable. Nevertheless, he questioned the horizon theory developed earlier and proposed that the ergosphere and not the horizon plays the main role in the electrodynamics. Uzdensky (2005) exploited the analogy with pulsar magnetospheres (Contopoulos, Kazanas \& Fendt 1999, hereafter CKF) and discussed a different possibility, namely the physical significance of the so called Light Surfaces (LS; see below). These are surfaces where magnetic field lines as a geometric construct 'rotate' at the speed of light in the same or the opposite direction with respect to ZAMOs. Quoting his work, '... one has to consider magnetic field lines that extend from the event horizon out to infinity. Since these field lines are not attached to a heavy infinitely conducting disk, their angular velocity $\omega$ cannot be explicitly prescribed; it becomes just as undetermined as the poloidal current I they carry. Fortunately, however, these field lines now have to cross two light surfaces (the inner one and the outer one). Since each of these is a singular surface of the force-free Grad-Shafranov equation, one can impose corresponding regularity conditions on these two surfaces. Thus, we propose that one should be able to devise an iterative scheme that uses the two light surface regularity conditions in a coordinated manner to determine the two free functions $\omega(\Psi)$ and $I(\Psi)$ simultaneously, as a part of the overall solution process... We realize of course that iterating with respect to two functions simultaneously may be a very difficult task....

Contopoulos, Papadopoulos \& Kazanas (2013, hereafter CPK) implemented precisely what Uzdensky set out to do a few years earlier and obtained the monopole 
solution using the LSs to self-consistently determine the unknown distributions of the magnetic field angular velocity and poloidal electric current for a split monopole force-free configuration. The astrophysical problem is, however, different since what we actually observe are jets and not an isotropic outflow. We, therefore, need something to collimate the jet. This agent may come in the form of a paraboloidal boundary along the wind from the surrounding accretion disk (e.g. Tchekhovskoy, Narayan \& McKinney 2010, hereafter TNM), or in the form of a background vertical magnetic field held in place by a disk far from the hole (e.g. Komissarov \& McKinney 2007; Palenzuela et al. 2010; Alic et al. 2012). In both cases, the collimated morphology of the outflow is dictated by the specific boundary conditions and not by the black hole itself.

In this paper we generalize the numerical method of CPK and improve its stability. Our solutions confirm and generalize the pioneering results of $\mathrm{BZ}$ and of previous time dependent numerical simulations for spin parameters up to maximal rotation and for various astrophysical boundary conditions. Our goal is a deeper understanding of the structure of the rotating black hole magnetosphere. In $\oint 2$ we re-derive the central equation of our problem, the general relativistic force-free Grad-Shafranov equation. In $\S 3$ we generalize the numerical approach of CPK and re-derive monopole and paraboloidal solutions. We also investigate a vertical magnetic field and show that this problem is degenerate (i.e. there exists an infinity of solutions). An interesting by-product of our numerical method is that we can also solve the electro-vacuum problem through the same equation. Finally, in $\S 4$ we discuss our results and show that, similarly to pulsars, black hole magnetospheres naturally develop an electric current sheet that potentially plays a very important role in the dissipation of black hole rotational energy and in the emission of high-energy radiation.

\section{BASIC EQUATIONS}

For completeness of the presentation, we will re-write the equations of steady-state axisymmetric force-free general relativistic electrodynamics presented in CPK since, as we argued, these are the physical conditions that pertain in the black hole magnetosphere. We assume that the background geometry is Kerr, and will work in Boyer-Lindquist coordinates $(t, r, \theta, \phi)$. We will use the ' $3+1$ ' formulation of MacDonald \& Thorne (1982). The metric is:

$$
d s^{2}=-\alpha^{2} d t^{2}+\varpi^{2}(d \phi-\Omega d t)^{2}+\frac{\Sigma}{\Delta} d r^{2}+\Sigma d \theta^{2},
$$

where,

$$
\begin{gathered}
\alpha=(\Delta \Sigma / A)^{1 / 2}, \\
\Omega=2 a M r / A, \\
\varpi=(A / \Sigma)^{1 / 2} \sin \theta, \\
\Sigma=r^{2}+a^{2} \cos ^{2} \theta, \\
\Delta=r^{2}-2 M r+a^{2}, \\
A=\left(r^{2}+a^{2}\right)^{2}-a^{2} \Delta \sin ^{2} \theta
\end{gathered}
$$

Here, $M$ is the mass of the black hole and $a$ its angular momentum $(0 \leq a \leq M), \alpha$ is the lapse function, $\Omega$ is the angular velocity of ZAMOs, and $\varpi$ is the cylindrical radius $(\varpi=r \sin \theta$ when $a=0)$. Through out this paper we use geometric units where $G=c=1$. $\mathbf{e}_{i}$ is the spatial coordinate basis and

$$
\mathbf{e}_{\hat{r}}=(\Delta / \Sigma)^{1 / 2} \mathbf{e}_{r}, \mathbf{e}_{\hat{\theta}}=(1 / \Sigma)^{1 / 2} \mathbf{e}_{\theta}, \mathbf{e}_{\hat{\phi}}=\frac{\Sigma}{A \sin \theta} \mathbf{e}_{\phi}
$$

are the unit basis vectors in the ZAMO frame. We consider steady-state, axisymetric, ideally conducting magnetospheres where $\mathbf{E} \cdot \mathbf{B}=0$. Under these assumptions, Maxwell's equations become

$$
\begin{gathered}
\nabla \cdot \mathbf{B}=0 \\
\nabla \cdot \mathbf{E}=4 \pi \rho_{e} \\
\nabla \times(\alpha \mathbf{B})=4 \pi \alpha \mathbf{J} \\
\nabla \times(\alpha \mathbf{E})=0,
\end{gathered}
$$

and the force-free condition yields

$$
\rho_{e} \mathbf{E}+\mathbf{J} \times \mathbf{B}=0 .
$$

Furthermore, the electric (E) and the magnetic $(\mathbf{B})$ field can be expressed in terms of three scalar functions $\Psi(r, \theta)$ (the total magnetic flux enclosed in the circular loop $r=$ const., $\theta=$ const. divided by $2 \pi), \omega(\Psi)$ (the angular velocity of the magnetic field lines), and $I(\Psi)$ (the poloidal electric current flowing through that loop), as

$$
\begin{gathered}
\mathbf{B}(r, \theta)=\frac{1}{\sqrt{A} \sin \theta}\left\{\Psi_{, \theta} \mathbf{e}_{\hat{r}}-\sqrt{\Delta} \Psi_{, r} \mathbf{e}_{\hat{\theta}}+\frac{2 I \sqrt{\Sigma}}{\alpha} \mathbf{e}_{\hat{\phi}}\right\}^{(5)} \\
\mathbf{E}(r, \theta)=\frac{\Omega-\omega}{\alpha \sqrt{\Sigma}}\left\{\sqrt{\Delta} \Psi_{, r} \mathbf{e}_{\hat{r}}+\Psi_{, \theta} \mathbf{e}_{\hat{\theta}}+0 \mathbf{e}_{\hat{\phi}}\right\}
\end{gathered}
$$

Putting all these together, eq. (44) can be transcribed as

$$
\begin{gathered}
\left\{\Psi_{, r r}+\frac{1}{\Delta} \Psi_{, \theta \theta}+\Psi_{, r}\left(\frac{A_{, r}}{A}-\frac{\Sigma_{, r}}{\Sigma}\right)-\frac{\Psi_{, \theta}}{\Delta} \frac{\cos \theta}{\sin \theta}\right\} \\
+\left[1-\frac{2 M r}{\Sigma}+\frac{4 M a \omega r \sin ^{2} \theta}{\Sigma}-\frac{\omega^{2} A \sin ^{2} \theta}{\Sigma}\right] \\
+\left(\frac{2 M r}{\Sigma}-\frac{4 M a \omega r \sin ^{2} \theta}{\Sigma}\right)\left(\frac{A_{, r}}{A}-\frac{1}{r}\right) \Psi_{, r} \\
+\left(\frac{\Sigma_{, r}}{\Sigma}-\frac{A_{, r}}{A}\right) \Psi_{, r} \\
-\left(2 \frac{\cos \theta}{\sin \theta}+\frac{A, \theta}{A}-\frac{\Sigma, \theta}{\Sigma}\right) \omega A(\omega-2 \Omega) \frac{\Psi_{, \theta} \sin ^{2} \theta}{\Delta \Sigma} \\
-2 \omega \Omega \varpi^{2} \frac{\Psi, \theta}{\Delta} \frac{A, \theta}{A}-2 M r \Sigma_{, \theta} \frac{\Psi_{, \theta}}{\Delta \Sigma^{2}} \\
-\frac{\omega^{\prime} A \sin ^{2} \theta}{\Sigma}(\omega-\Omega)\left(\Psi_{, r}^{2}+\frac{1}{\Delta} \Psi_{, \theta}^{2}\right) \\
=-\frac{4 \Sigma}{\Delta} I I^{\prime},
\end{gathered}
$$

the general relativistic force-free Grad-Shafranov equation. $(\ldots)_{, i}$ denotes partial differentiation with respect 
to $i$, whereas $(\ldots)^{\prime}$ denotes differentiation with respect to $\Psi$. Notice that if we set $M=a=0$ we obtain the standard pulsar equation in flat spacetime (Sharlemann \& Wagoner 1973). The zeroing of the term multiplying the second order derivatives in the previous equation, namely

$$
1-\frac{2 M r}{\Sigma}+\frac{4 M a \omega r \sin ^{2} \theta}{\Sigma}-\frac{\omega^{2} A \sin ^{2} \theta}{\Sigma}=0,
$$

yields the singular surfaces of the problem, the so called Light Surfaces (LSs) where

$$
\alpha^{-1}(\omega-\Omega) \varpi= \pm 1
$$

in units where $c=1$ (MacDonald \& Thorne 1982). One can interpret the expression in the left hand side of the above equation as the corotation velocity of magnetic field lines (as a geometric construct) with respect to ZAMOs. Outside the Outer Light Surface (OLS), magnetic field lines rotate faster than the speed of light with respect to ZAMOs, while inside the Inner Light Surface (ILS) they also rotate faster than the speed of light but in the opposite direction to that of ZAMOs. The ILS lies between the horizon and the static limit (the boundary of the ergosphere), whereas the OLS is asymptotically cylindrical (see Appendix B). In the case of pulsars, eq. (8) yields the standard pulsar light cylinder $\omega r \sin \theta=1$. We remind the reader of the 'natural radiation condition' at infinity (energy must flow outwards along all field lines), namely

$$
0 \leq \omega \leq \Omega_{\mathrm{BH}}
$$

(BZ). $\Omega_{\mathrm{BH}}=a / 2 M r_{\mathrm{BH}}$ is the angular velocity at the event horizon $r_{\mathrm{BH}}=M+\sqrt{M^{2}-a^{2}}$. We would also like to note here that in an electro-vacuum (no charges, no currents) one solves the equation

$$
\boldsymbol{\nabla} \times(\alpha \mathbf{B})=0 .
$$

The reader can easily check that the above expression is equivalent to eq. (7) if we set $\omega=\Omega$ and $I=0$. In other words, we can solve the electro-vacuum problem by using the same numerical code that we have developed to solve the general relativistic Grad-Shafranov equation. It is interesting that the factor multiplying the second order term of the equation never becomes zero in electrovacuum.

Equation (7) is a second order elliptic equation for $\Psi(r, \theta)$ with singular surfaces, and can be solved numerically with boundary conditions that correspond to various astrophysical systems. Our numerical method is described in detail in CPK. We repeat here its main elements which are essentially the same as the ones implemented by CKF when they obtained the first solution of the axisymmetric pulsar magnetosphere. Our goal is to obtain a magnetic field configuration where field lines pass smoothly through two singular surfaces while at the same time satisfying the specific boundary conditions of the astrophysical problem. There are two free functions in the problem, $\omega(\Psi)$ and $I(\Psi)$, that we can freely adjust in order to attain our goal. In other words, the solution will break down at the singular surfaces, except for one particular choice of $\omega(\Psi)$ and $I(\Psi)$. Our approach to determine these two functions is the following: we iteratively evolve the solution everywhere with a standard relaxation method for elliptic equations, and every few relaxation steps we adjust the two free functions by taking into consideration how much the field lines are 'broken' at the two singular surfaces. We find it helpful to correct $\omega(\Psi)$ at the inner light surface and $I(\Psi)$ at the outer one. We do not have a theory that will determine the number of relaxation steps one must wait before updating $\omega$ and $I$, nor how much one must correct them. This is done empirically with specific correction weights that depend on the numerical grid resolution. As in CPK, we changed the radial variable from $r$ to $R(r) \equiv r /(r+M)$ in order to extend our numerical integration from the event horizon $r_{\mathrm{BH}}$ which corresponds to $R_{\min } \equiv R\left(r_{\mathrm{BH}}\right)$, to some maximum radial distance $r_{\max }$ which corresponds to $R_{\max }=R\left(r_{\max }\right)$. The $\theta$ coordinate extends from the axis of symmetry $\left(\theta_{\min }=0\right)$ to the equatorial plane $\left(\theta_{\max }=\pi / 2\right)$. We implemented a $200 \times 64$ numerical grid uniform in $R$ and $\theta$. Notice that this grid has a very high resolution in $r$ near the event horizon where the ILS lies, but not as high around the OLS, and in particular at low $\theta$ and $a$ values. At small $\theta$ 's we are, therefore, obliged to use expansions for $\Psi \propto \sin ^{2} \theta$ (limit of eq. 17), $\omega \rightarrow 0.5 \Omega_{\mathrm{BH}}$ (limit of eq. 12 below when the field configuration around the axis is monopolar), and $I \rightarrow 0$.

We have found that the horizon boundary condition is not important since it is an 'inner infinity', and as long as the numerical relaxation proceeds 'smoothly', the horizon regularity condition

$$
I(\Psi)=-0.5\left(\Omega_{\mathrm{BH}}-\omega\right) \frac{\sqrt{A}}{\Sigma} \Psi_{, \theta} \sin \theta
$$

(Znajek 1977) is automatically satisfied. The outer (radial infinity) boundary condition is similarly not important 2 (see Appendix A). We discretize all physical quantities and we update $\Psi(R, \theta)$ through simultaneous overrelaxation with Chebyshev acceleration (subroutine SOR from Numerical Recipes; Press, Flannery \& Teukolsky 1986). The final solution does not depend on our particular choice of initial magnetic field configuration.

We update the distributions of $\omega(\Psi)$ and $I(\Psi)$ as follows: At each latitude $\theta$, we check where the singularity condition (eq. 8) is satisfied in $r$. At each such radial position, we extrapolate $\Psi$ inwards from larger $r\left(\Psi\left(r^{+}, \theta\right)\right)$ and outwards from smaller $r\left(\Psi\left(r^{-}, \theta\right)\right)$ using the adjacent three grid points along $r$. In general, $\Psi\left(r^{+}, \theta\right)$ and $\Psi\left(r^{-}, \theta\right)$ differ. Then, at the ILS we implement

$$
\begin{aligned}
\omega_{\text {new }}\left(\Psi_{\text {new }}\right)= & \omega_{\text {old }}\left(\Psi_{\text {new }}\right) \\
& -\mu_{\omega}\left[\Psi\left(r^{+}, \theta\right)-\Psi\left(r^{-}, \theta\right)\right],
\end{aligned}
$$

whereas at the OLS we implement

$$
\begin{aligned}
I I_{\text {new }}^{\prime}\left(\Psi_{\text {new }}\right)= & I I_{\text {old }}^{\prime}\left(\Psi_{\text {new }}\right) \\
& +\mu_{I I^{\prime}}\left[\Psi\left(r^{+}, \theta\right)-\Psi\left(r^{-}, \theta\right)\right],
\end{aligned}
$$

where

$$
\Psi_{\text {new }} \equiv 0.5\left[\Psi\left(r^{+}, \theta\right)+\Psi\left(r^{-}, \theta\right)\right]
$$

2 In an astrophysical elliptic problem, the boundary conditions at infinity do not have any effect on the solution near the origin, as was the case in CKF. However, this is not true when one implements boundary conditions in computational domains of finite spatial extent, as was the case in Ogura \& Kojima (2003) where the effect of the boundaries is easily discerned in their figure 1 . 


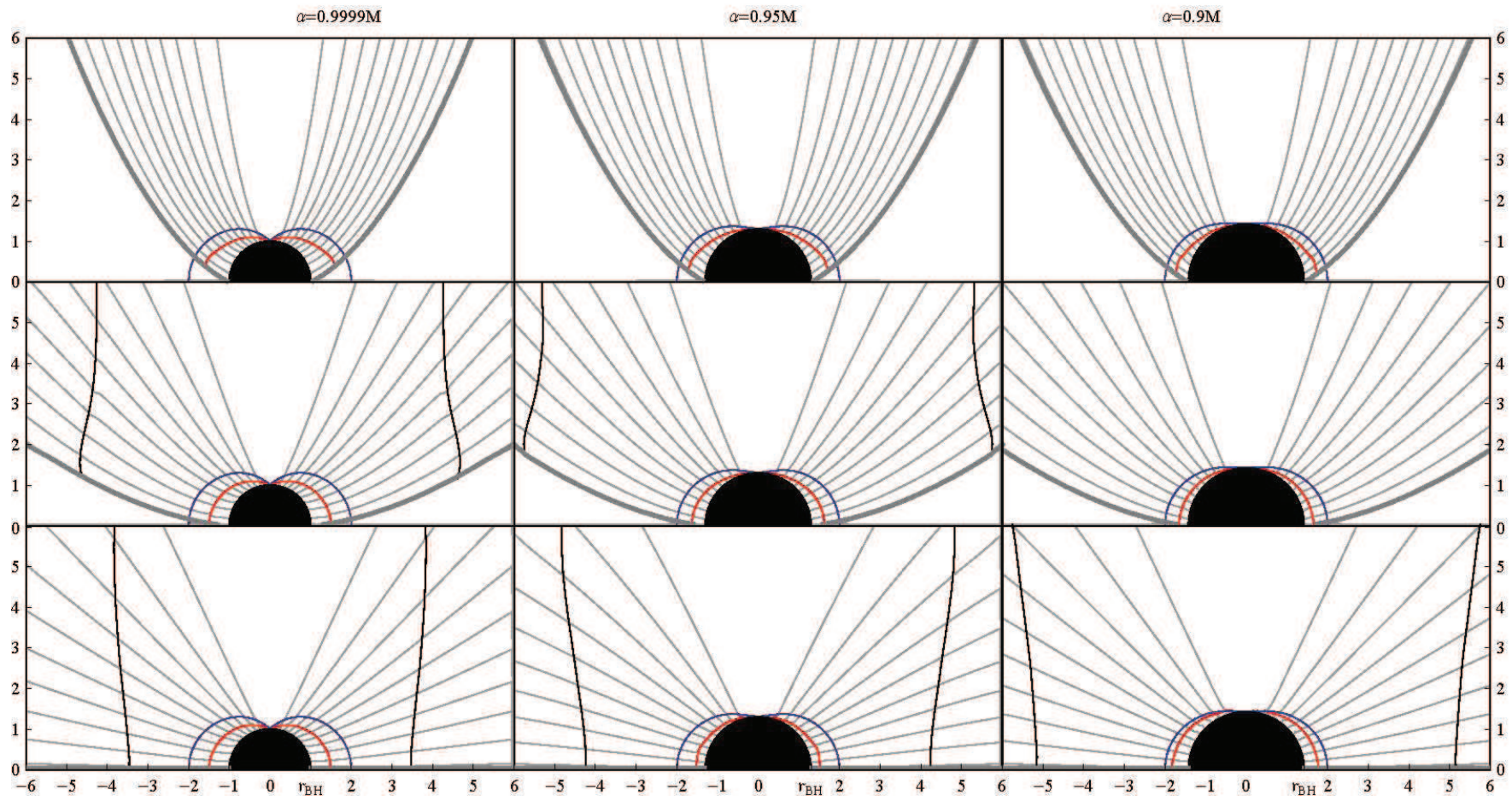

Figure 1. Monopole and paraboloidal solutions. $\nu=1$. Top to bottom: $r_{0}=1,10, \infty$. Left to right: $a=0.9999 M, 0.95 M, 0.9 M$. Black semi-circle: the horizon. Red/blue/black lines: the ILS, the static limit, and the OLS respectively. Where not seen, the OLS lies outside the region shown. Thick grey line: collimating boundary / equatorial disk. 
at each LS. The reasoning here is that we impose weighted corrections on $\omega$ and $I$ based on the nonsmoothness of $\Psi(r, \theta)$ along the two LS. As we said above, the weight factors $\mu_{\omega}$ and $\mu_{I I^{\prime}}$ are obtained empirically and depend on the grid resolution. Notice that we adjust $I I^{\prime}$ and not directly $I$ since it is $I I^{\prime}$ that appears as a source term in the right hand side of eq. (77). $I(\Psi)$ is then obtainable through direct numerical integration, namely $I(\Psi)=\left[2 \int_{0}^{\Psi} I I^{\prime}(\psi) \mathrm{d} \psi\right]^{1 / 2}$. This is a very general procedure that may be applied to any similar singular equation. The new element with respect to $\mathrm{CPK}$ is that once the values of $\omega\left(\Psi_{\text {new }}\right)$ and $I I^{\prime}\left(\Psi_{\text {new }}\right)$ are updated, we fit a polynomial of order 5 in $\Psi_{\text {new }}$ for $\omega$, and of order 9 for $I I^{\prime}$. The choice of polynomial order is determined empirically. The zeroth order term in $\omega$ is set equal to $0.5 \Omega_{\mathrm{BH}}$, whereas the zeroth order term in $I I^{\prime}$ is set equal to zero. All these improvements made the numerical scheme much more stable than the one implemented in CPK. Once the relaxation method starts, numerical convergence proceeds without obstacles. For each value of $a$ and for particular boundary conditions, this procedure yields a single set of angular velocity and electric current distributions and a unique solution that crosses smoothly the two singular surfaces.

\section{SOLUTIONS}

\subsection{Monopole}

As we discussed in CPK, contrary to pulsars, the source of the black hole magnetic field lies outside the event horizon in the surrounding distribution of matter (accretion disk, torus, disk wind, etc.). Therefore, the study of the black hole magnetosphere necessarily involves the exterior boundary conditions of the specific astrophysical problem that we are considering, i.e. there is no such thing as an isolated black hole magnetosphere. In that respect the pulsar magnetosphere is a 'cleaner' problem.

The simplest magnetized black hole scenario with astrophysical interest is one where the electric currents supporting its magnetic field are distributed on a thin equatorial disk that extends all the way to the horizon. We have found out that the outer boundary condition is unimportant (as long as the solution extends smoothly to infinity and fills all space). We implemented the following boundary conditions on a numerical grid $\left(R_{\min } \leq R \leq 1,0 \leq \theta \leq \pi / 2\right)$ :

$$
\begin{gathered}
\Psi(R, \theta=0)=0, \\
\Psi\left(R, \theta=\frac{\pi}{2}\right)=\Psi_{\mathrm{BH}},
\end{gathered}
$$

where, $\Psi_{\mathrm{BH}}$ is the total magnetic flux threading the black hole horizon. We initialize our numerical grid with the Michel (1982) split monopole, namely

$$
\Psi(r, \theta)=\Psi_{\mathrm{BH}}(1-\cos \theta)
$$

with

$$
\begin{gathered}
I(\Psi)=-0.5 \omega(\Psi) \Psi\left(2-\frac{\Psi}{\Psi_{\mathrm{BH}}}\right), \text { and } \\
\omega(\Psi)=0.5 \Omega_{\mathrm{BH}} .
\end{gathered}
$$

We also tried different initializations (e.g. paraboloidal) and the numerical method converged to the same solu- tion. Notice that eq. (18) is an exact solution for the flat or Schwarzschild spacetime of a pulsar where one can assume that the magnetic field angular velocity is constant for all field lines. For a slowly rotating black hole with small $a$, the solution is very close to the split monopole as shown by BZ. There is a practical problem with low $a$ 's though: the event horizon is very close to the boundary of the ergosphere, therefore, the numerical scheme requires a very high radial resolution in order to treat the effect of the ILS that lies between the two. At the same time, the OLS is very far away (remember that it is analogous to the light cylinder of a slowly rotating pulsar), and therefore, our numerical procedure is not adequate to treat such a problem. Our procedure works best for high black hole spin parameters where the ILS is sufficiently distanced from the event horizon, and the OLS is not too far away.

As our numerical integration proceeds, both distributions $\Psi(r, \theta)$ and $\omega(\Psi)$ evolve, and therefore, the shape and position of the ILS and the OLS also evolve. In other words, a certain magnetic field line characterized by a certain value of $\Psi$ will cross each singular surface in a different place. After about $3000-4000$ iterations the angular velocity and electric current distributions relax to a steady state that allow the magnetic flux function $\Psi$ to pass smoothly through the two LS. The numerical procedure is stable, contrary to CPK where it was hard to reach an unchanging asymptotic solution. We show results for three values of $a=0.9999 M, 0.95 M$ and $0.9 M$. As the black hole become maximally rotating the magnetic flux becomes concentrated along the axis of symmetry $\theta=0$. Our solutions directly reproduce and confirm the results of previous time dependent numerical simulations (e.g. TNM).

The obtained electric current distribution is implemented physically through an outflow of electrons beyond the OLS that is connected to an inflow of positrons inside the ILS all the way to the event horizon. We will not discuss the origin of these particles and we can only refer the interested reader to discussions of the wind generation zone in the literature (Komissarov 2004b, Globus \& Levinson 2013). What is most interesting, though, is that, similarly to pulsars, in this model too $I\left(\Psi_{\mathrm{BH}}\right) \neq 0$, and as a result, the poloidal electric circuit needs to close in the form of a current sheet along the last open magnetic field line that crosses the black hole horizon. Therefore, the magnetospheric current sheet seems to be a generic feature of black hole magnetospheres, and as is the case with pulsars, this is expected to be associated with the origin of high energy radiation ( see $\S 4$ ).

Another obvious result is that equatorial boundary conditions do not produce any collimated energy outflow that would resemble an astrophysical jet. In other words, as in pulsars, black hole magnetospheres do not naturally produce jets. Nevertheless, jets are observed in nature, and therefore, what is needed is some collimating agent that would restrict the lateral expansion of the magnetosphere. This can be found in the form of a disk wind or a thick torus configuration surrounding the magnetic flux that threads the black hole horizon.

\subsection{Paraboloidal}




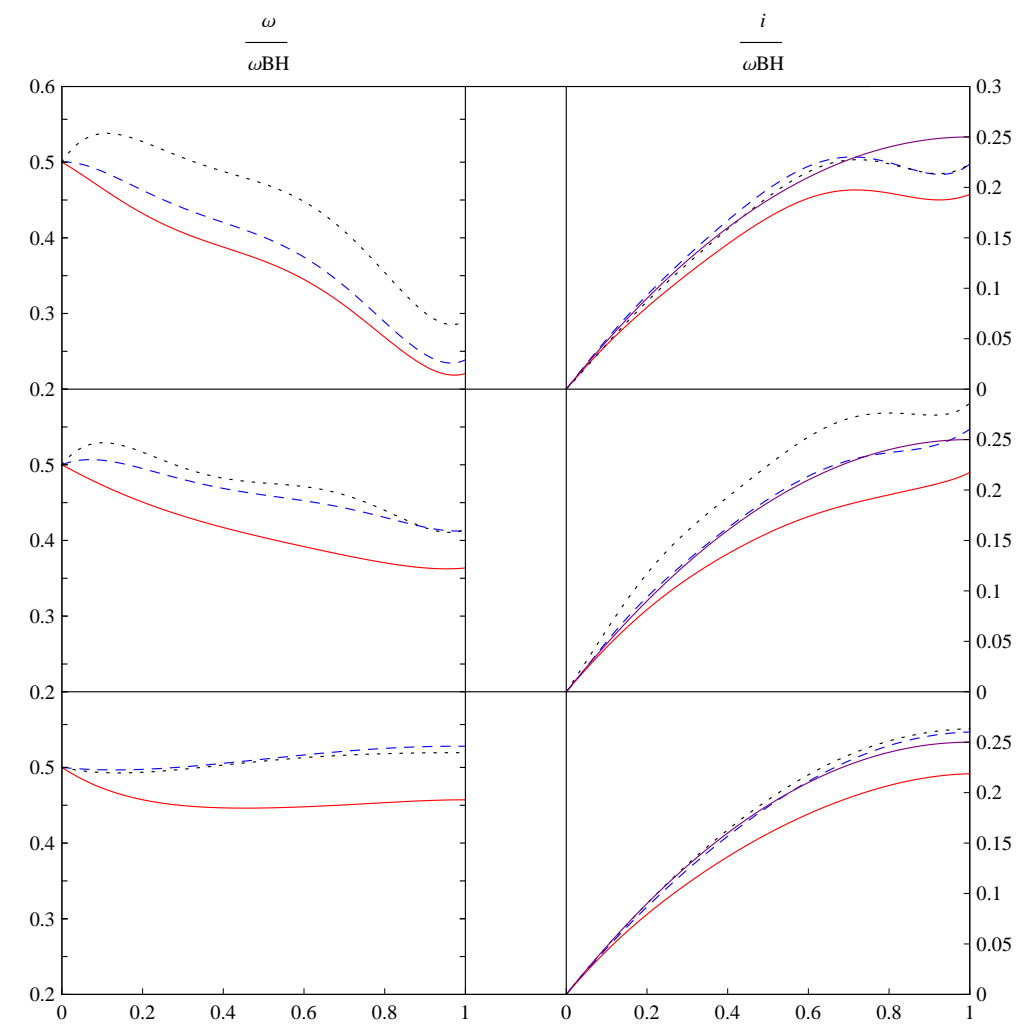

Figure 2. Left column: distribution of magnetic field angular velocity $\omega(\Psi) / \Omega_{\mathrm{BH}}$ over $\Psi / \Psi_{\mathrm{BH}}$ for various values of $a$. Right column: distribution of electric current $I(\Psi) / \Omega_{\mathrm{BH}} \Psi_{\mathrm{BH}}$. Solid/dashed/short-dashed lines: $a=0.9999 M, 0.95 M$ and $0.9 M$ respectively. Purple line: Michel's monopole solution in flat spacetime. Top to bottom: $r_{0}=1,10, \infty . \nu=1$.

Following TNM we considered a paraboloidal wall described by

$$
1-\cos \theta_{\mathrm{wall}}=\left(\frac{r+r_{0}}{r_{\mathrm{BH}}+r_{0}}\right)^{-\nu} .
$$

as a boundary condition that would yield collimated solutions. $\nu$ is just a shape parameter. Notice that the smaller $r_{0}$ the more collimated the boundary. As in the monopolar case, the wall intersects the event horizon at the equator. We implemented the following boundary conditions on a numerical grid $\left(R_{\min } \leq R \leq R_{\max }, 0 \leq\right.$ $\theta \leq \pi / 2)$ :

$$
\begin{aligned}
\Psi(R, \theta=0) & =0, \\
\Psi\left(R, \theta \geq \theta_{\text {wall }}(r)\right) & =\Psi_{\mathrm{BH}}, \\
\Psi_{, R}\left(R=R_{\text {max }}, \theta<\theta_{\text {wall }}\left(r_{\max }\right)\right. & =0 .
\end{aligned}
$$

The reason we cannot really extend our numerical integration to $R=1$ is that we used the same numerical grid that we used in the monopole case, and solved for $\Psi$ only for angles $\theta \leq \theta_{\text {wall }}(r)$. The grid resolution becomes worse and worse as we move higher up along the paraboloidal wall, and at some distance that corresponds to about $r_{\max }=13 M$, our numerical iteration at the OLS breaks down. A better numerical approach would have been to re-write our equations in a paraboloidal grid $(0 \leq R \leq 1,0 \leq \Theta \leq 1)$ where $\Theta \equiv \theta / \theta_{\text {wall }}(r)$. We initialize our numerical grid with:

$$
\Psi(r, \theta)=\Psi_{\mathrm{BH}}\left(\frac{r+r_{0}}{r_{\mathrm{BH}}+r_{0}}\right)^{\nu}(1-\cos \theta) .
$$

The split monopole is a special case of the above configuration with $r_{0}=\infty$ or $\nu=0$.

Our numerical integration showed that the angular velocity changes dramatically from the monopole one, going from $0.5 \Omega_{\mathrm{BH}}$ on the axis to about $0.3 \Omega_{\mathrm{BH}}$ near the wall. One can see in the figures how the outer light surface deforms because of the change in the distribution of the magnetic field angular velocity. As described previously, all paraboloidal solutions contain a return poloidal electric current sheet that now flows along the wall that collimates the jet. Similarly to the monopole configuration, this too is expected to be associated with the origin of high energy radiation from a collimated black hole magnetosphere.

\subsection{Vertical magnetic field}

We next consider the case of a black hole embedded in a vertical magnetic field. This is obviously an artificial scenario that has nonetheless been considered by many previous research groups (Palenzuela et al. 2010, Komissarov \& McKinney 2007 ). The electro-vacuum case has been first studied by Wald (1974), where it was shown that a maximally rotating black hole expels the magnetic flux from the vicinity of the event horizon (King, Lasota \& Kundt 1975). This has been named the black hole 'Meissner effect' (in analogy to superconductors) in the literature (Komissarov \& McKinney 2007). We too can easily reproduce the electro-vacuum solutions. For $a=0.9999 M$ the magnetic flux is almost totally expelled from the event horizon, but as we move to lower spin parameter values more and more flux passes through the event horizon.

The force-free case is more interesting. We imple- 


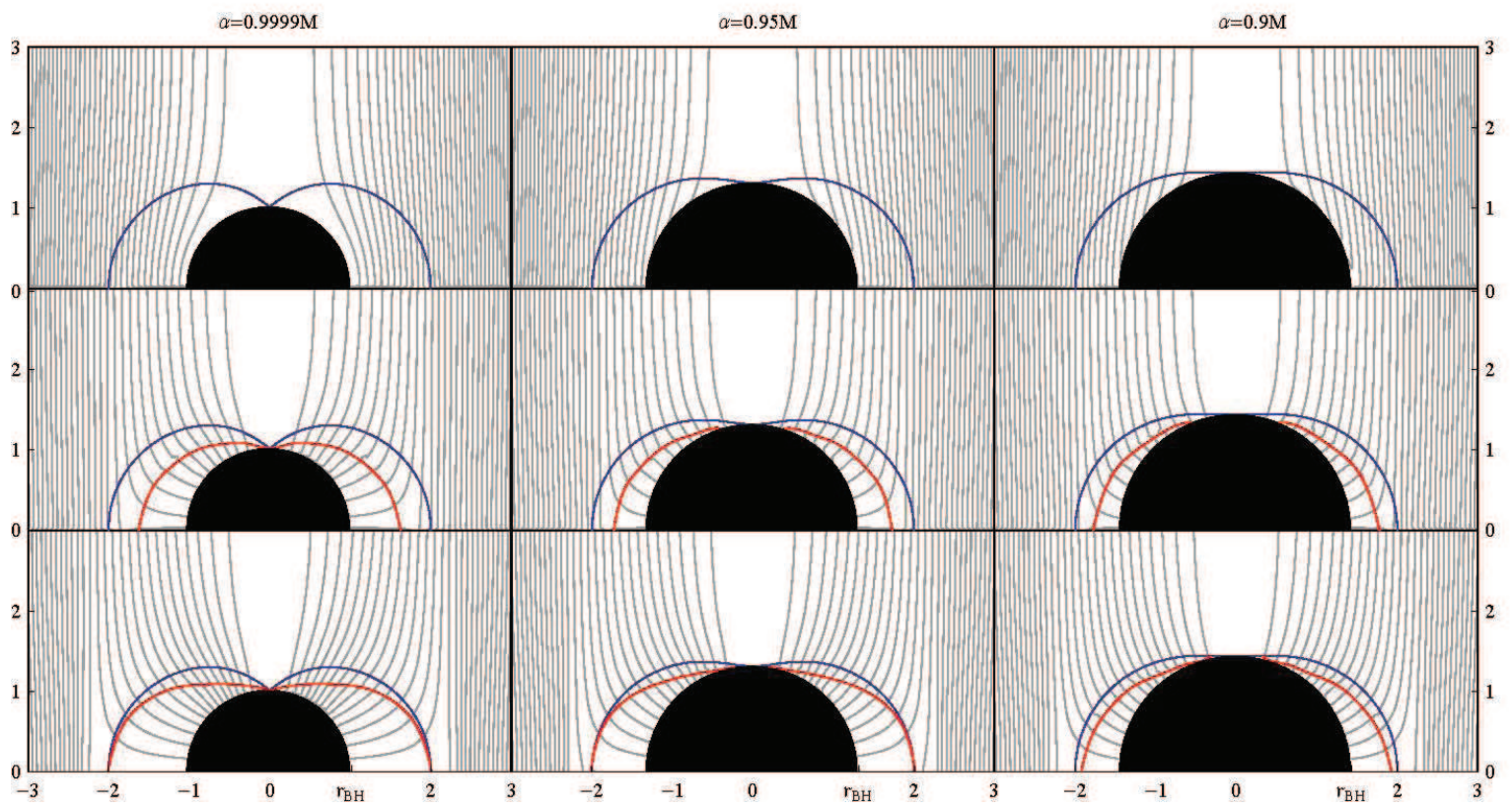

Figure 3. Rotating black hole embedded in a vertical magnetic field for various imposed distributions of $\omega(\Psi)$. Line colors as in fig. 1 . Top row: electrovacuum. Second row: $\omega(\Psi)$ given by eq. (27). Notice the presence of field lines that pass through the ergosphere but do not cross the ILS, thus they do not carry any electric current, nor any electromagentic (Poynting) flux. Notice also the development of a poloidal electric current sheet along the last field line that crosses the black hole horizon. Third row: $\omega(\Psi)$ given by eq. (26). Notice the suppression of the 'Meissner' effect under force-free conditions. 
mented the following boundary conditions on a numerical grid $\left(R_{\min } \leq R \leq 1,0 \leq \theta \leq \pi / 2\right)$ :

$$
\begin{gathered}
\Psi(R, \theta=0)=0, \\
\Psi(R, \theta)=\Psi_{\max }\left(\frac{r \sin \theta}{r_{\mathrm{BH}}}\right)^{2} \text { for } r \sin \theta \geq 4 M, \\
\Psi_{, R}(R=1, \theta)=0,
\end{gathered}
$$

where $\Psi_{\max }$ is some canonical value for the magnetic flux. Here too, we used the same numerical grid that we used in the monopole case, and solved for $\Psi$ only for cylindrical radii $r \sin \theta \leq 4 M$. We initialize our numerical grid with a uniform vertical field

$$
\Psi(r, \theta)=\Psi_{\max }\left(\frac{r \sin \theta}{r_{\mathrm{BH}}}\right)^{2},
$$

One can easily see that there is a problem. Some magnetic field lines cross the ILS, but none crosses both the ILS and OLS. Therefore, we cannot implement the previous numerical procedure where both $\omega(\Psi)$ and $I(\Psi)$ are determined through the condition of smooth crossing of both LSs. We thus implement a different approach:

It is well known that no field lines can cross the same LS twice (Gralla \& Jacobson 2014). This is exactly analogous to what happens in the pulsar magnetosphere where closed stellar field lines that cross the light cylinder open up to infinity because matter tied to those field lines cannot corrotate. The same applies here. Field lines that cross the ILS open from inside and are stretched all the way to 'inner infinity', i.e. the event horizon. We do not know a priori which field lines will cross the ILS, and which will not. As we evolve our numerical relaxation, the system pulls some flux toward the event horizon, and an ILS forms. The lines that do not cross the ILS cross the equator vertically $\left(\Psi_{, \theta}(r, \pi / 2)=0\right)$. Obviously, because of north-south symmetry, no electric current can flow along such field lines, and therefore $I I^{\prime}(\Psi)$ must equal zero along them. The ones that do cross the ILS, though, are brought to the event horizon through an equatorial boundary condition of horizontal field along the equator between the horizon and the edge of the ILS inside the ergosphere. This obviously corresponds to an electric current sheet which, in order to guarantee electric circuit closure, continues along the last field line that crosses the horizon to infinity. This must be implemented manually as in CKF, otherwise the force-free Grad-Shafranov equation treats it just as a discontinuity in $I I^{\prime}$ and entirely misses its effect 3 . The presence of this current sheet is evident through the discontinuity in the azimuthal component of the magnetic field $B_{\phi}$, which imposes an equivalent discontinuity in the poloidal field (in order to guarantee continuity of $B^{2}-E^{2}$ ) across the last field line that crosses the black hole horizon. The poloidal field discontinuity can be discerned in the solutions presented in Figure 3. As we said the solution passes only from one LS, so we cannot determine both

\footnotetext{
3 In practice, we set $I(\Psi)=I\left(\Psi_{\mathrm{BH}}\right) \exp \left[-\left(\Psi-\Psi_{\mathrm{BH}}\right)^{2} / 2 \sigma^{2}\right]$ for $\Psi>\Psi_{\mathrm{BH}}$, and $\sigma$ is an arbitrary parameter that characterizes the effective current sheet width. $\sigma$ must be chosen as small as practically possible given a particular numerical grid resolution. Notice that if we do not implement this current sheet, the solution will be different, as was the case in Ogura \& Kojima 2003.
}

$\omega(\Psi)$ and $I(\Psi)$. In other words, the solution is degenerate.

In practice, what we did was to arbitrarily specify a certain distribution for the angular velocity $\omega(\Psi)$ and then find the unique electric current distribution $I(\Psi)$ that allows a smooth solution through the ILS. We obtained typical results for

$$
\begin{aligned}
& \omega=0.5 \Omega_{\mathrm{BH}} \cos ^{2}\left(\frac{\pi}{2} \frac{\Psi}{\Psi_{\mathrm{BH}}}\right), \\
& \omega=0.5 \Omega_{\mathrm{BH}}\left(1-\frac{\Psi}{\Psi_{\mathrm{BH}}}\right)^{2}
\end{aligned}
$$

etc., and $\omega=0$ for $\Psi>\Psi_{\mathrm{BH}}$. $\Psi_{\mathrm{BH}}$, the maximum magnetic flux that threads the horizon, evolves as the numerical relaxation progresses. In these cases the ILS meets the boundary of the ergosphere at the equator, and all field lines entering the ergosphere are rotating and are filled with electric current that contributes to the total electromagnetic output of the solution. This choice is arbitrary. We realized that we don't need to require $\omega(0)=0.5 \Omega_{\mathrm{BH}}$ along the axis when the field configuration is not monopolar there. We tried arbitrary values of $\omega(0)$ (e.g. $\left.0.8 \Omega_{\mathrm{BH}}, 0.3 \Omega_{\mathrm{BH}}\right)$ and still obtained smooth solutions, each one for a different current distribution. The same applies on the equator. The angular velocity does not have to go to zero there. We thus also solved the equation for different distributions like

$$
\omega=\Omega_{\mathrm{BH}}\left(0.5-\frac{\Psi}{\Psi_{\mathrm{BH}}} 0.25\right)
$$

etc. For the latter distribution, $\omega$ reaches $0.25 \Omega_{\mathrm{BH}}$ at the equator (other values work equally well), and therefore, the ILS ends inside ergosphere. Field lines that do not cross the ILS and pass through the ergosphere do rotate but they don't carry any electric current $(I(\Psi)=0)$ so they don't take part in the electromagnetic output of the solution. The angular velocity of these field lines is set to fall gradually to zero with radial distance, in a way that none of them accidentally crosses any LS. Moreover, these field lines cross the equator vertically, and one can easily check (using eqs. 5, 6] and 8) that, along the equator, $B^{2}-E^{2}=\left(B^{\theta}\right)^{2}-\left(E^{r}\right)^{2}>0$ everywhere outside the ILS, and $B^{2}-E^{2}=0$ at the point where the ILS crosses the equator. This supports our previous conclusion that no field lines that cross the ILS can cross the equator vertically inside the ILS $\left(B^{2}-E^{2}\right.$ would become negative there). Instead, such field lines are stretched all the way to the event horizon, forming the equatorial current sheet that we described above. Therefore, energy and angular momentum are extracted along all magnetic field lines that penetrate the ILS, even when the ILS lies completely inside the ergosphere, as is the case shown in the middle row of Figure 3. This result generalizes the discussion in Section 6.1.2 of Komissarov (2004a).

There is still interest in the so called 'Meissner effect' because of the possibility that it 'could quench jet power at high spins (Pena 2014). We believe that in a force-free plasma filled environment (i.e. electron-positron plasma) no Meissner effect will ever occur. As we discussed previously, if somehow magnetic flux is brought close to the horizon field lines passing the ILS twice will open from the inside and will be naturally stretched all the way to 
the horizon. Therefore, for a high spin black hole, magnetic flux will not be expelled from the horizon. Instead, the system will bring flux to the horizon. This process is fully supported by our solutions of a maximally rotating black hole embedded in a force-free vertical magnetic field.

\section{DISCUSSION}

Blandford \& Znajek (1977) estimated that typical astrophyscial magnetic fields will naturally break down the vacuum around astrophysical black holes and will establish a force-free magnetosphere. In the last decade general relativistic magnetohydrodynamic simulations were performed to explore the BZ mechanism and its relation with jet observations (McKinney \& Gammie 2004, TNM, Sadowski et. al. 2013). What we learn from these simulations is that in the region close to the polar axis and near the black hole the plasma density becomes so low that artificial corrections must be implemented (matter density and energy 'flloors') in order to keep the simulations running. This suggests that the system wants to impose force-free conditions. In other words, force-free is a good approximation in these regions.

For the case of a black hole embedded in a vertical magnetic field we have shown that we can have infinitely many solutions for each value of $a$. A particular limit of that problem, one with a uniform field at infinity, has been addressed by several research groups through time dependent simulations (Komissarov \& McKinney 2007; Palenzuela et al. 2010; Alic et al. 2012). These simulations are shown to converge to a unique (for each code) distribution for the angular velocity and electric current. In this particular case, the condition of a uniform vertical field at infinity imposes one extra condition between $\omega$ and $I$, namely $I=-\omega \Psi$ (eq. (35); Appendix A), and therefore, $\omega$ and $I$ are uniquely defined. Furthermore, $\omega(\Psi)=0$ along field lines that do not thread the black hole horizon, since $I(\Psi)=0$ along those field lines. As a result, both $\omega(\Psi)$ and $I(\Psi)$ must reach zero along the last field line $\Psi_{\mathrm{BH}}$ that threads the horizon, and therefore, the ILS reaches the static limit along the equator, and the poloidal electric current sheet disappears. We plan to address this problem in a future work, were we will also perform a systematic comparison between our results and the results of previous numerical simulations.

Another reasonable question is what is the astrophysical significance (if any) of such solutions (a black hole embedded in a vertical magnetic field). Magnetic field lines are generated by electric currents in a surrounding distribution of matter, and therefore, the vertical extent of the vertical field region cannot extend beyond 'a few times' the radial extent of the inner edge of that distribution of matter. Magnetic field lines must close, and when closure is taken into consideration, an OLS will develop which will uniquely constrain the solution. Another problem arises when people consider vertical magnetic fields in the problem of two merging black holes. In that case, the spiralling black holes empty a region of matter one or two orders of magnitude more extended than their respective gravitational radii. Correspondingly, the magnetic field that can be held there by the surrounding matter distribution is at least one order of magnitude smaller than the canonical value that would correspond to a field held by an accretion disk that ex- tends down to the ISCO of the black hole. In that case, the electromagnetic power is in reality at least two orders of magnitude smaller than estimates obtained in the literature (see Lyutikov 2010 for details). We conclude that vertical magnetic field configurations are artificial and such solutions do not correspond to real astrophysical jets.

A very important element in all our solutions is the poloidal current sheet that is naturally formed, where the electric circuit closes. The current sheet lies along the equatorial thin disk in the monopole solutions, and along the boundary wall as we pass to the paraboloidal solutions. As in pulsars, high energy radiation is expected from reconnection processes that result in particle acceleration along these current sheets (Lyubarsky \& Kirk 2001; Li, Spitkovsky \& Tchekhovskoy 2012; Kalapotharakos et al. 2012). For the monopole and paraboloidal solutions this implies that high energy radiation may not be coming along the axis of rotation but in a direction orthogonal to it, as in the orthogonal gamma-ray burst model of Contopoulos, Nathanail \& Pugliese (2014).

We are also interested in the power output of our solutions which we can directly calculate once we obtain the corresponding distribution of $\omega(\Psi)$ and $I(\Psi)$ as

$$
P=2 \int_{0}^{\Psi_{\mathrm{BH}}} \omega(\psi) I(\psi) d \psi .
$$

In all cases, $P \approx \Omega_{\mathrm{BH}}^{2} \Psi_{\mathrm{BH}}^{2}$. One sees directly that the total magnetic flux $\Psi_{\mathrm{BH}}$ accumulated through the horizon and the black hole angular velocity $\Omega_{\mathrm{BH}}$ contribute together to the power output of the black hole. Most people focus only on the role of the black hole spin, but the issue of the flux accumulation remains equally (and probably even more) important. This straightforward result has sometimes been overlooked in the debate on whether a jet power-black hole spin association is observable in X-ray binaries black hole jets (Steiner, Narayan \& McClintock 2013, Russell et al. 2013). It may also account for the famous AGN radio loud / radio quiet dichotomy (Sikora et al. 2007). The efficiency of the flux accumulation close to the black hole may be related either to the efficiency of flux advection (e.g. Sikora \& Begelman 2013), or our favorite, the in situ flux generation by the Poynting-Robertson Cosmic Battery effect (Contopoulos \& Kazanas 1998).

Finally, a comment on the extent of the accretion disk is in order. In all our monopole and paraboloidal solutions the accretion disk extends all the way to the horizon. This may not be true in reality. The disk holding the magnetic flux may end several gravitational radii away from the black hole. As an extension of our work we would like to investigate such solutions. We expect that, because the accretion disk will stop before reaching the horizon, magnetic field lines will cross the ILS twice, thus they will open from inside and will be stretched all the way to the 'inner infinity', the event horizon. Another interesting scenario that we would like to investigate is one where the accretion disk is starting to disperse leaving behind a rotating magnetospheric charge of finite radial extent. This will correspond to a ring current that will generate its own dipole magnetic field (as in Lyutikov 2012). At large distances, such configurations will 
be similar to pulsar magnetospheres.

We thank the referee, Pr. Serguei Komissarov, for his constructive comments and criticism. This work was supported by the General Secretariat for Research and Technology of Greece and the European Social Fund in the framework of Action 'Excellence'.

\section{REFERENCES}

Alic, D., Moesta, P., Rezzolla, L., Zanotti, O. \& Jaramillo, J. L. 2012, ApJ, 754, 36

Blandford, R. D. \& Znajek, R. L. 1977, MNRAS, 179, 433 (BZ77)

Contopoulos, I. 2005, A\& A, 442, 579

Contopoulos, I. \& Kazanas, D. 1998, ApJ, 508, 859

Contopoulos, I., Kazanas, D. \& Fendt, C. 1999, ApJ, 511, 351

Contopoulos, I., Kazanas, D. \& Papadopoulos, D.B. 2013, ApJ,765113

Gralla, S. E. \& Jacobson, T. 2014, arXiv:1401,6159

Globus, N. \& Levinson, A. 2013 Phys.Rev. D, 88, 4046

Kalapotharakos, C., Harding, A. K., Kazanas, D.\& Contopoulos, I. 2012, ApJ, 754, 1

King, A. R., Lasota, J. P. \& Kundt, W. 1975, PThPh, 12, 3037

Komissarov, S. S. 2001, MNRAS, 326, 41

Komissarov, S. S. 2004, MNRAS, 350, 427 (a)

Komissarov, S. S. 2004, MNRAS, 350, 1431 (b)

Komissarov, S. S. \& McKinney, J. C. 2007, MNRAS, 377, L49
Lasota, J. P., Gourgoulhon, E., Abramowicz, M., Tchekhovskoy, A. \& Narayan, R. 2014 Phys.Rev. D, 89, 024041

Li, J., Spitkovsky, A. \& Tchekhovskoy, A.2012,ApJ, 746, 60

Lyubarsky, Y. \& Kirk, J. G. 2001, ApJ, 547,437

Lyutikov, M. 2010, arXiv:1010.6254

Lyutikov, M. 2012, arXiv:1209.3785

MacDonald, D. A. \& Thorne, K. S. 1982, MNRAS, 198, 345

McKinney, J. C., Gammie, C. F. 2004, ApJ, 611, 977

Michel, F. C. 1982, Rev. Mod. Phys., 54, 1

Narayan, R. \& McClintock, J. E. 2012, MNRAS, 419, 69

Ogura, J. \& Kojima, Y. 2003, PThPh, 109, 619

Palenzuela, C., Garrett, T., Lehner, L. \& Liebling, S. L. 2010, Phys. Rev. D, 82, 044045

Pena, R. 2014, arXiv:1403.0938

Press, W. H., Flannery, B. P., Teukolsky, S. A. 1986, 'Numerical recipes. The art of scientific computing', Cambridhe Univ. Press

Punsly, B. \& Coroniti, F. V. 1990, ApJ, 354, 583

Russell, D. M., Gallo,E. \& Fender, R. P. 2013, MNRAS, 431, 405

Sadowski, A., Narayan, R., Pena, R. \& Zhu, Y. 2013, MNRAS, 436, 3856

Scharlemann, E. T. \& Wagoner, R. V. 1973, ApJ, 182, 951

Sikora, M. \& Begelman, M. C. 2013, ApJ, 764, L24

Sikora, M., Stawarz, L., Lasota, J. P. 2007, ApJ, 658, 815

Tchekhovskoy, A., Narayan, R. \& McKinney, J. C. 2010, ApJ, 711,50

Thorne, K. S., Price, R. H. \& MacDonald D. A. Black Holes: The Membrane Paradigm Yale University Press 1986

Uzdensky, D. A. 2005, ApJ, 620, 889

Wald, R. M. 1974, Phys. Rev. D, 10, 1680

Znajek, R. L. 1977, MNRAS, 179, 457

\section{APPENDIX A: THE REGULARITY CONDITIONS AT 'INFINITY'}

The horizon regularity condition was first derived by Znajek (1977) and was used as a boundary condition in the perturbative solution obtained in Blandford \& Znajek (1977). MacDonald \& Thorne (1982) derived it in their formalism and applied it in the 'membrane paradigm' (Thorne et al. 1986).

Our understanding of the event horizon is that it is an 'inner infinity' similar to the normal ('outer') infinity. Any electromagnetic field configuration that is generated by a finite (spatially) distribution of electric currents and extends from the horizon to infinity $r \rightarrow \infty$ with both $r \sin \theta$ and $r \cos \theta \rightarrow \infty$ along every field line satisfies the radiation condition $E=B$ there (this is not the case for a vertical magnetic field), with $\mathbf{E} \times \mathbf{B}$ pointing in the direction of 'infinity'. Under steady-state axisymmetric force-free conditions in particular,

$$
E^{\theta}= \pm B^{\phi} \text { at 'infinity' }
$$

(from eqs. [5 and 6). At the outer infinity the plus sign applies and the above equation yields

$$
I(\Psi)=-0.5 \omega(\Psi) \Psi_{, \theta} \sin \theta
$$

It is straightforward to show that, when we multiply eq. (30) with its $\Psi$ derivative, we obtain

$$
4 I I^{\prime}=\left(\omega^{2} \Psi_{, \theta \theta}+\omega^{2} \frac{\cos \theta}{\sin \theta} \Psi_{, \theta}+\omega \omega^{\prime} \Psi_{, \theta}^{2}\right) \sin ^{2} \theta,
$$

which is just eq. (7) in the limit $r \rightarrow \infty$ when $r^{2} \Psi_{, r r}$ and $r \Psi_{, r}$ are much smaller than both $\Psi_{, \theta \theta}$ and $\Psi_{, \theta}$ (along any field line that extends to infinity, $\Psi \rightarrow$ const. and $r \Psi_{, r} \rightarrow 0$, because otherwise, if $r \Psi_{, r} \rightarrow a \neq 0$, that would imply $\Psi \rightarrow a \ln r+$ const., and therefore $a=0$ ). Similarly, at the inner infinity (the black hole horizon) the minus sign applies and eq. (29) yields

$$
I(\Psi)=-0.5\left(\Omega_{\mathrm{BH}}-\omega(\Psi)\right) \frac{\sqrt{A}}{\Sigma} \Psi_{, \theta} \sin \theta,
$$

the Znajek condition (eq. 12). Here too it is straightforward (albeit more tedious) to show that, when we multiply eq. (32) with its $\Psi$ derivative, we obtain

$$
4 I I^{\prime}=\left(\left(\omega-\Omega_{\mathrm{BH}}\right)^{2} \Psi_{, \theta \theta}+\left(\omega-\Omega_{\mathrm{BH}}\right)^{2} \frac{\cos \theta}{\sin \theta} \Psi_{, \theta}-\frac{\Sigma_{, \theta}}{\Sigma} \Psi_{, \theta}+\omega^{\prime}\left(\omega-\Omega_{\mathrm{BH}}\right) \Psi_{, \theta}^{2}\right) \frac{A}{\Sigma^{2}} \sin ^{2} \theta,
$$

which too is just eq. (7) in the limit $r \rightarrow r_{\mathrm{BH}}$ with $\Delta \rightarrow 0$.

We have thus shown that the two 'infinity' conditions do not teach us anything about the $\omega(\Psi)$ nor the $I(\Psi)$ of astrophysical solutions. These are determined from the two LSs. Any 'smooth' astrophysical solution that extends all the way from the horizon to infinity with both $r \sin \theta$ and $r \cos \theta \rightarrow \infty$ along every field line adjusts $\Psi_{, \theta}$ so as to satisfy both equations. We did check that this is indeed the case in all our solutions to within $0.5 \%$. Obviously, the 
other boundary conditions (along the equator, or the surrounding collimating boundary) and the requirement that the magnetosphere fills all space are also important in determining the solution that corresponds to the particular astrophysical problem under consideration. The same holds true in pulsars where the solution there is fully determined by the light cylinder 1 .

Notice that the vertical field configuration also satisfies eqs. (32) \& (33) but not eqs. (30) \& (31). At infinity, the problem becomes one of special relativity, and the vertical field configuration is better described through the generalized pulsar equation in cylindrical coordinates $\varpi \equiv r \sin \theta$ and $z \equiv r \cos \theta$, namely

$$
4 I I^{\prime}=\omega^{2} \varpi^{2}\left(\Psi_{, \varpi \varpi}+\Psi_{, \varpi} / \varpi\right)+\omega \omega^{\prime} \varpi^{2} \Psi_{, \varpi}^{2}-\left(\Psi_{, \varpi \varpi}-\Psi_{, \varpi} / \varpi\right)
$$

(eq. 10, Contopoulos 2005). It is interesting that, in the particular limit of a uniform vertical magnetic field at infinity, $\Psi \propto \varpi^{2}$ and eq. (34) yields $I I^{\prime}=\omega^{2} \Psi+\omega \omega^{\prime} \Psi^{2}$ and therefore

$$
I=-\omega \Psi .
$$

\section{APPENDIX B: LIGHT SURFACES}

We generated Figure 4 by solving eq. (8) for constant $\omega$ because we want the reader to appreciate how the Light Surfaces (LSs) change shape as the spin of the black hole and the magnetic field angular velocity change. We are not the first to discuss the role of LSs. They were introduced from the very beginning by Blandford \& Znajek (1977), and a nice mathematical description can be found in Komissarov (2004a). For $\omega=0$ the ILS meets the static limit, the boundary of the ergosphere. For $\omega=\Omega_{\mathrm{BH}}$ the inner light surface meets the event horizon.

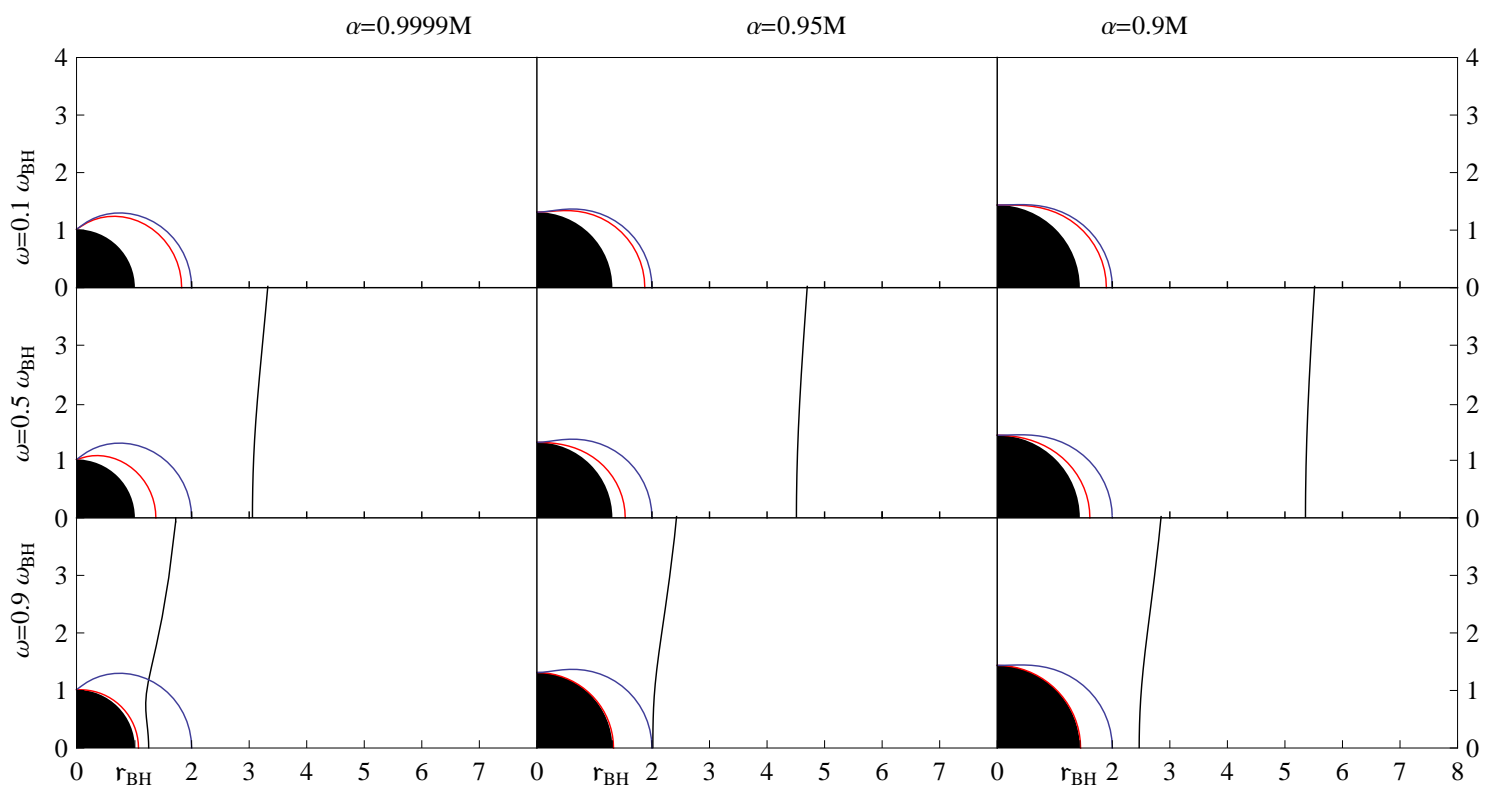

Figure 4. Light Surfaces for various black hole spin parameters $a$ and magnetic field angular velocities $\omega$. Line colors as in fig. 1.

${ }^{4}$ Pulsars have one extra boundary condition, namely that their magnetosphere originates on the stellar surface. 